\title{
Multiple Processes for Least Mean Square Adaptive Algorithm on Roadway Noise Cancelling
}

\author{
Sri Arttini Dwi Prasetyowati*, Adhi Susanto** \\ *Dep. of Electrical Engineering, Industrial Technology Faculty, Sultan Agung Islamic University, Semarang, Indonesia \\ ** Dep. of Electrical Engineering, Faculty of Engineering, Gadjah Mada University, Yogyakarta, Indonesia
}

\section{Article Info}

\section{Article history:}

Received Jan 4, 2014

Revised Feb 22, 2015

Accepted Mar 10, 2015

\section{Keyword:}

Adaptive process

Filter length

LMS adaptive

Noise cancelling

Vehicle's noise

\begin{abstract}
Noise is a problem often found in daily life. Noise also make people could not concentrate to do their work. Efforts to reduce noise have been proposed, but, due to variety of the noise's characteristics, every noise problem requires different solution. This research aim to cancel the vehicle's noise while maintaining the information heard. These conditions happened in the hospitals classrooms, or work room near the roadway. The vehicle's noise changes very fast, so the adaptive system is the good solution candidate for solving this problem. On the beginning, the simulation process had the trouble with the iterations. Matlab software only can execute the certain range of iteration. It could not cancel the noise, even the information becomes criptic. The problem is how to cancell the vehicle's noise with the restriction software and still manage the important information. This research will modify the LMS adaptive algorithm so that the iteration could be done by the system and the main goal of the research could be reached. The modification of the algorithm is based on the filter length (L) used to adapt with the noise. Therefore, this research conducted simulation of the adaptive noise cancelling with two process steps. The output of the first adaptive process have the.same characteristics with the noise that would be cancelled, thus the first adaptive process have the error near to zero. The second adaptive process changes the input by the output of the first process and mix the information into the noise. Error occured in the final process is the information heard as the dominant output.
\end{abstract}

Copyright $(2015$ Institute of Advanced Engineering and Science. All rights reserved.

\section{Corresponding Author:}

Sri Arttini Dwi Prasetyowati,

Departement.of Electrical Engineering,

Industrial Technology Faculty

Sultan Agung Islamic University, Semarang, Indonesia

Email: arttini@unissula.ac.id

\section{INTRODUCTION}

This research is inspired by actual situation in a hospital room in Semarang contaminated with noises from the nearby road. It is very difficult to make conversations between administration officers and guests in the presence of roadway noises, thus the officers need to frequently repeat the information. Therefore, it is of great importance to find a technique to cancel the noise while the information can still be heard.

The vehicle noise changes fast, hence the system must adapt speedily and accurately. Least Mean Square (LMS) Adaptive is a prospective solution for this problem due to its robustness; moreover the algorithm doesn't need many known variables. Adaptive system is system that can adapt (without operator) so that it can be optimal. The system always does the process so that the variable is very close to the target [1]. Adaptive Noise Cancellation (ANC) application is useful in wide range of scenarios, such as conference room [2]. It means that the adaptive algorithm is very suitable for research on roadway noise cancellation. 
In fact, if the target fluctuates over time (not static), the process should be done as soon as possible while the error $\varepsilon$ must be kept minimal. Adaptive systems have several characteristics: can adapt automatically, can be trained to do filtering and make decision, have simpler steps than the non-adaptive systems although have a complex analysis, and can develop the model [1]. LMS algorithms have performance issues related to insufficient excitation, nonstationary reference inputs, finite precision arithmetic, quantization noise, and measurement noise. Such factors cause weight drift and potential instability in the conventional LMS algorithm [3]. Besides LMS algorithms, there are several adaptive algorithms, e.g. NLMS (Normalized Least Mean Square), RLS (Recursive Least Square). One of possible schemes is to place an identical filter in the reference signal path to the weight update of the LMS algorithm, also called Filtered-x LMS (FxLMS) algorithm [4].

Using the simplest structure that have an easy explanation for the analytic description, Linear Combiner, the LMS Adaptive can be executed simply. The formula can be shown as:

$$
y_{k}=w_{o k} x_{k}+w_{1 k} x_{k-1}+w_{2 k} x_{k-2}+. .+w_{L k} x_{k-L}=\sum_{l=0}^{L} w_{l k} x_{k-l}
$$

Output $y_{k}$ can be calculated from the linear combination of the input $x_{k}$ and the weight $w_{l k}$. LMS Adaptive is one of the simplest Adaptive Algorithm that can solve the complex problem of vehicle's noise. The LMS Adaptive Algorithm can be shown on the equation (2).

$$
\mathbf{W}_{\mathbf{k}+\mathbf{1}}=\mathbf{W}_{\mathbf{k}}+2 \mu \mathbf{X}_{k} \varepsilon_{k}
$$

Equation (2) is used to find the correct weight used in Equation (1). In addition, the Mean Square Error (MSE) needs to be determined, mainly from the difference of noise to be cancelled and the output of the system. Equations (1) and (2) are used in the configuration described in Figure 1 for vehicle noise cancelling in this work.

Figure 1 showed configuration with two input, the first input contains signal $s_{k}$ and noise $n_{k}$, while the other input contains only noise signal $n_{k}^{\prime}$, under the condition $n_{k}^{\prime}$ and $n_{k}$ come from same noise but taken from different places. In block "S.A", the input and output are processed with Linear Combiner, whereas in block "Algoritma" there is process for finding the weight. Adaptive algorithm adjusts the filter coefficient included in the vector $W_{n}$. The adaptive filter aims to equate its output $\mathrm{y}(\mathrm{n})$ to the desired output $d(n)$. For each iteration, the error signal is given by:

$$
\varepsilon(n)=d(n)-y(n)
$$

where $\varepsilon$ or error is difference between desired input $\mathrm{d}$ and output $\mathrm{y}$. The error signal is fed back into the filter, where the filter characteristics are altered accordingly as shown in Figure 2 [5].

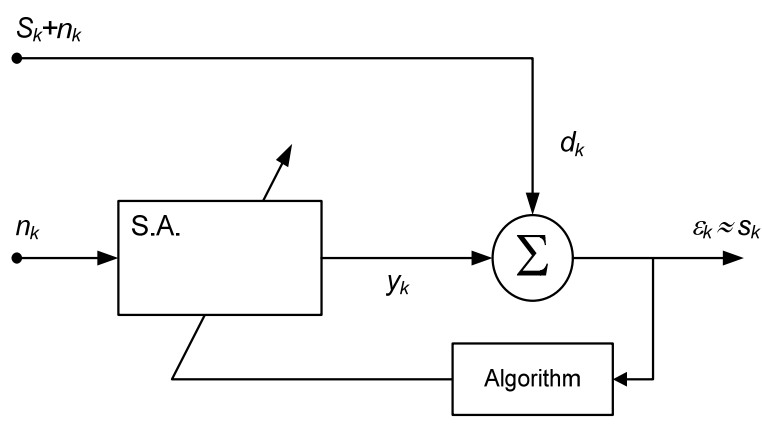

Figure 1. Configuration of Noise Cancelling

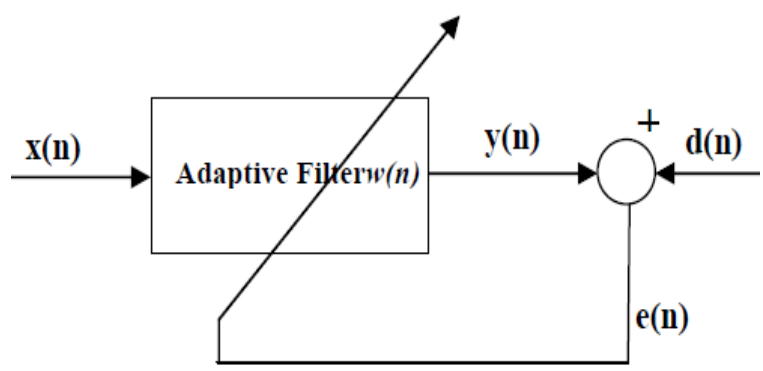

Figure 2. Adaptive Filter Configuration 


\section{SEARCHING OPTIMUM DELAY VALUE AND TWO PROCESS LMS ADAPTIVE NOISE CANCELLING}

Data Collection was done by recording vehicle noise from the different location in the same time. The first noise $\left(n_{k}\right)$ in Figure 1 is vehicle noise heard in the room designated for the noise canceling. The second noise is the roadway noise $\left(n_{k}^{\prime}\right)$ from the source of the noise. The noise comes from motorcycle and cars with different fuel types. Furthermore, information signal was also recorded and simulated combined with the first roadway noise $\left(s_{k}\right)$. It should be noted that the first and the second recording were done simultaneously. Simple tools that could help the recording as a divider of the two data recording were added on computer as shown in Figure 3.

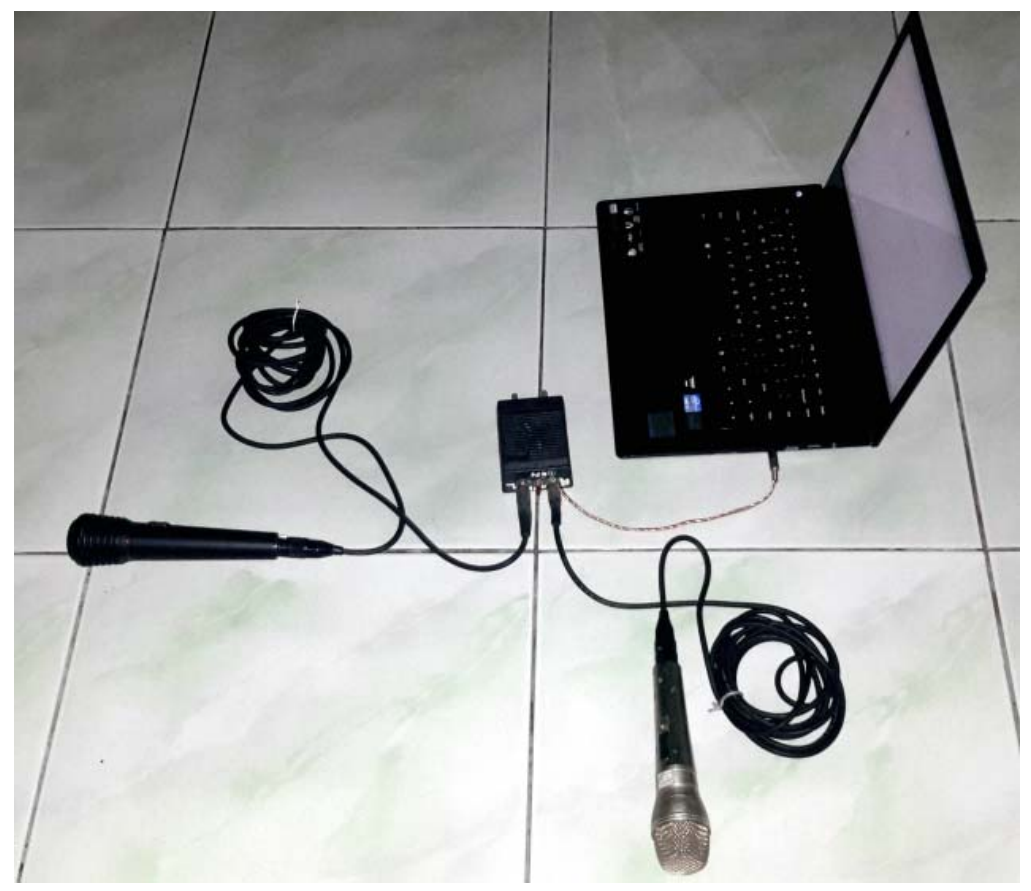

Figure 3. Simple tool divider the two noise signal

Searching the optimum delay value was done for five vehicle noise sources, i.e. truck, bus, motorcycle, car with solar fuel, car with non solar fuel, and mix of several vehicles. Because of the difficulty of computing, the optimum iteration was selected for noise cancelling. The input of Adaptive LMS is the noise heard in the room (the first noise) and the reference of the Adaptive LMS is noise from the source (the second noise). The first step was looking for the optimum delay with $\mu=0,001$ and determining the amount of iteration due to number of sample 20.000. Searching of the optimum delay based on moving average from the square error $\left(\varepsilon^{2}\right)$.

By observing the value of the delay, increasing it and changing $L$ value periodically, optimum value of delay and L could be met. The larger the $\mathrm{L}$ value taken, the mean from moving average tend to convergent and less value can be obtained. Ideally, each vehicle's noise has different $\mathrm{L}$ and delay. In fact the value that most influential in LMS Adaptive process is the value of $\mu$. Table 1 show the value of Delay and $\mathrm{L}$ with the same $\mu$ for all vehicles' noise. According to the obtained data, Delay and L value can be added or subtracted according to the required computation [6].

It can be seen that the minimum value for $\mathrm{L}$ is 150 , and the maximum value for $\mathrm{L}$ is 450 . For the weight iteration taken 20.000 , it will be hard for the computation, because algorithm will look for the output with 150 iteration each which would be processed untill 20.000 iterations for searching the weights. Consequently, it takes considerably long time. From the difficulty of searching L value and the big iterations for finding the weights, this research divides the LMS adaptive noise cancelling into two states in hope to get fast process and minimal error in result.

Figure 4 show the two processes LMS adaptive noise cancelling. The process stages are: 
- First Process: $d$ is indoor Signal (SD), $x$ is the reference of the adaptive LMS or outdoor signal (SL), to be expected the output close to SD, so that the error value $\varepsilon$ (error) close to zero.

- Second Process: $d$ is SD mixed by information signal, whereas $x$ is taken from output from the first process. To be expected error value $\varepsilon$ close to information signal [7].

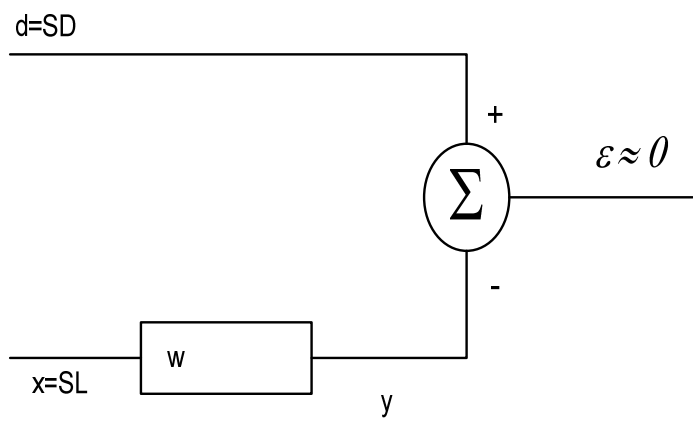

(a)

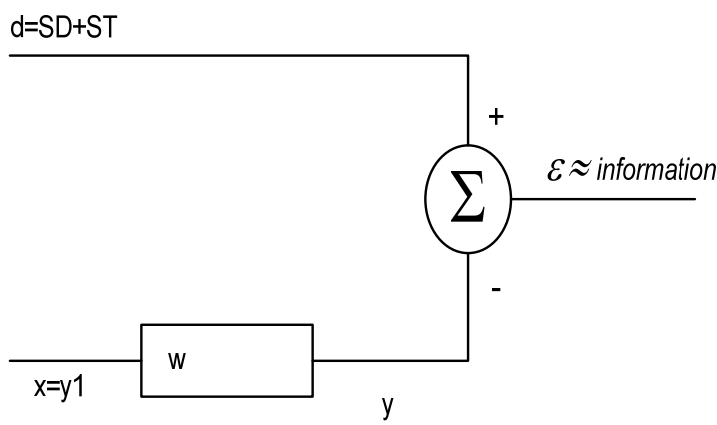

(b)

Figure 4. The two processes LMS adaptive noise cancelling; (a) The first process of LMS adaptive algorithm, (b) The second process of LMS adaptive algorithm

The Mean Square Error (MSE) for LMS Adaptive with only one process and two process were obtained by determining the $\mathrm{L}$ value on the state position, that eventually $\mu$ and delay change. The $\mathrm{L}$ value is optimum value that can be taken in the LMS Adaptive Process, so that the computational simulation can be solved. However, if more powerfull computing resource are present, L value does not need to be taken minimal, but at any value that could achieve the smallest MSE.

\section{RESULTS AND DISCUSSION}

The Process of calculating $\mathrm{L}$ and delay with the diesel-based vehicles as a noise signal using "Moving Average" methods and $\mu=0,001$, is shown in Figure 5 and Figure 6.

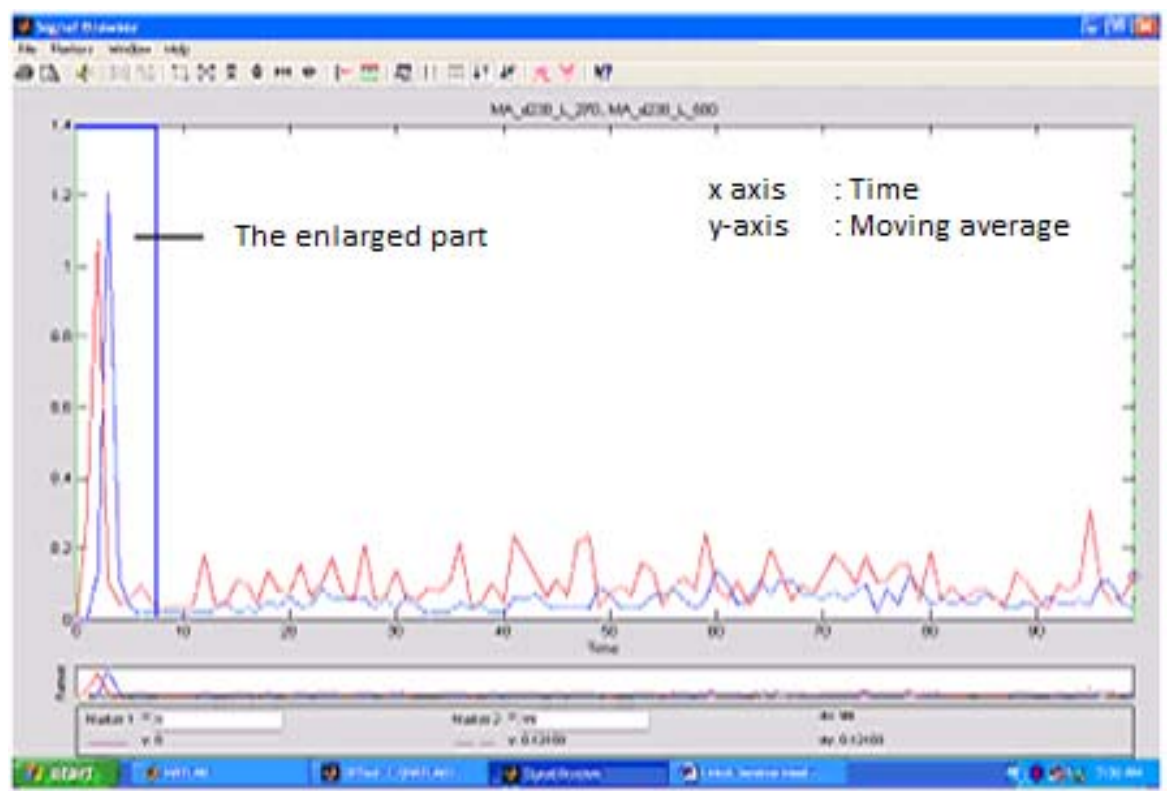

Figure 5. The Process of calculating L and delay; $\mathrm{L}=190$ delay 220 (red), $\mathrm{L}=200$ delay 230 (blue), $\mathrm{L}=220$ delay 230 (green). 


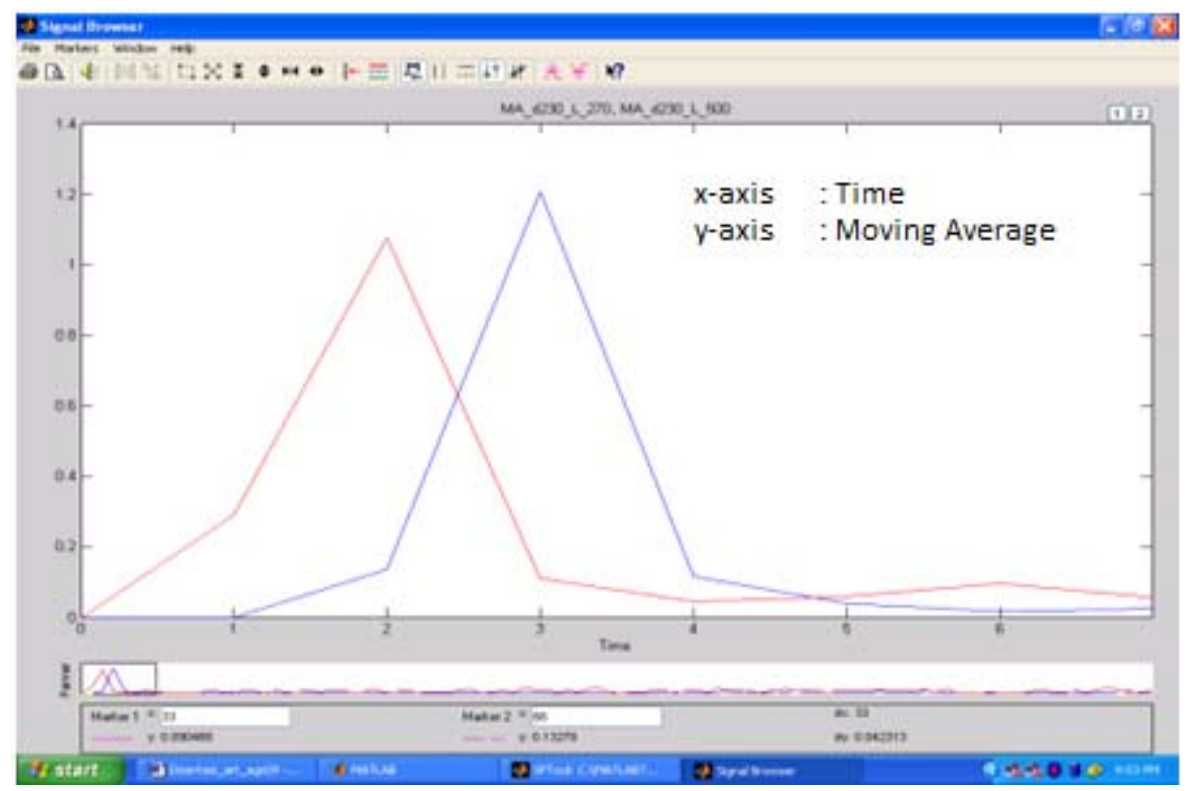

Figure 6. The Process of calculating L and delay; enlarged part in the first peak of the Figure 1.

It could be concluded from figure 5 and 6 that the bigger the value of $\mathrm{L}$ the square moving averge is more convergent. However, as a consequence, the computation is also getting harder, and the process becomes longer. Nevertheless, it does not guarantee that the computation gives accurate results. The Optimal delay with 230 samples of 0.0052 second is a good value. However to prevent the missing of delay, the delay was taken on 200 samples. The result for the other vehicles for 20.000 iterations and $\mu=0,001$, are shown in Tabel 1. From Tabel 1 showed that L value very high for some vehicles. The bigger value of $L$ the noise will be erased completely because the value of moving average convergent to certain number. However the process becomes so hard. However, to anticipate the big value of L, LMS adaptive algorithms were modified into two processes.

Tabel 1 . The value of $\mathrm{L}, \mu$, and delay for several vehicles

\begin{tabular}{lccc}
\hline Vehicles & $\begin{array}{c}\mathbf{L} \\
\text { (Iteration) }\end{array}$ & $\begin{array}{c}\mu \\
\text { (Fixed) }\end{array}$ & $\begin{array}{c}\text { Delay } \\
\text { (sample) }\end{array}$ \\
\hline Diesel & 150 & 0.001 & 200 \\
Bus & 250 & 0.001 & 200 \\
Truck & 350 & 0.001 & 200 \\
Car & 450 & 0.001 & 200 \\
Motor & 250 & 0.001 & 200 \\
Car with Solar Fuel & 300 & 0.001 & 200 \\
\hline
\end{tabular}

The MSE value obtained from LMS adaptive algorithm on the first and second processes was investigated and subsequently compared with the L value in one process. Table 2 and 3 show the MSE values for several vehicles in the first and second process of LMS Adaptive, respectively. The $\mu$ value was determined constant and small enough, so that the process is running slowly but quite thorough.

Tabel 2. The MSE values of car with solar fuel in the first process LMS Adaptive

\begin{tabular}{cccc}
\hline $\mathbf{L}$ & $\begin{array}{c}\text { Delay } \\
\text { (Iteration) }\end{array}$ & $\begin{array}{c}\boldsymbol{\mu} \\
\text { (Sample) }\end{array}$ & MSE1 \\
\hline 100 & 230 & 0,001 & 10.2357 \\
230 & 110 & 0,001 & 0,1131 \\
230 & 100 & 0,001 & 0,1200 \\
230 & 120 & 0,001 & 0,1041 \\
230 & 130 & 0,001 & 0,1041 \\
230 & 140 & 0,001 & 0,1049 \\
\hline
\end{tabular}


Table 3. The MSE values of car with solar fuel in the second process LMS Adaptive

\begin{tabular}{ccccc}
\hline $\begin{array}{c}\mathbf{L} \\
\text { (Iteration) }\end{array}$ & $\begin{array}{c}\text { Delay } \\
\text { (Sample) }\end{array}$ & $\begin{array}{c}\mu \\
\text { (Fixed) }\end{array}$ & MSE1 & MSE2 \\
\hline 100 & 230 & 0,001 & 0,2400 & 16,2944 \\
230 & 100 & 0,001 & 0,0998 & 3,9117 \\
230 & 110 & 0,001 & 0,0990 & 11,6033 \\
230 & 120 & 0,001 & 0,0995 & 7,0464 \\
230 & 130 & 0,001 & 0,1008 & 32,7102 \\
\hline
\end{tabular}

Table 2 and 3 reveal that $\mu$ is selected with constant value while $\mathrm{L}$ and delay vary. The $\mathrm{L}$ value is the optimal value that can reach in the LMS Adaptive process, because the computer has many limitations in its calculation [6]. The first process has MSE1 less than 1, and this is a small value because in the first process there is no information mix in the input. The second process has MSE2 more than 1 because the MSE2 include information that must be occurred as an error.

\section{CONCLUSION}

From the research experiments, the roadway noise cancelling could be realized with LMS Adaptive Algorithm with modification into two processes, with $\mathrm{L}=230, \mu=0,001$, and delay as much as 100 samples with linear combiner structure. With one process of LMS adaptive, the L value is still cannot cancell the vehicle's noise because the process need big L value, but with two process of LMS Adaptive, the bigger L can be reached, therefore the noise cancelling could be realized well.

\section{REFERENCES}

[1] Widrow, B., and S.D. Stearns, "Adaptive Signal Processing”, 1985, Prentice-Hall, Inc., Englewood Clifts, New Jersey.

[2] Scarpiniti, M., Daniel, C., Raffaele, P., Aurelio, U, "A Collabarative Filter Approach to Adaptive Noise Cancellation”, Smart Innovation, Systems, and Technologies, Volume 19, 2013, pp 101-109.

[3] David A. Cartes, Laura R. Ray, and Robert D.Collier, "Lyapunov Tuning of The Leaky LMS Algorithm For Single Source", Single-Point-Noise Cancellation, Proceedings of the American Control Conference Arlington, VA June $25-27,2001$

[4] Sanaullah, Khan, M. Arif, T. Majeed, "Comparison of LMS, RLS and Notch Based Adaptive Algorithms for Noise Cancellation of a typical Industrial Workroom”, IEEE, 2004

[5] Akingbade KF, Isiaka AJA, "Separation of Digital Audio Signal Using Least-Mean-Square (LMS) Adaptive Algorithm", International Journal of Electrical and Computer Engineering (IJECE), Vol.4, No.4, August 2014, pp.557-560, ISSN: 2088-8708

[6] Prasetyowati, S.A.D, "Exploration of The Adaptive Vehicle's Noise Cancellation in The Work Room", Disertation, Gadjah Mada University, Yogyakarta, Indonesia, 2010

[7] Prasetyowati, S.A.D., Bustanul, A., Eka N.B.S., "Solution for Vehicles Noise Cancellation with Modification of LMS Adaptive Algorithm", International Journal on Computer Science and Engineering, Vol: 4 Issue: 5, Engg Journal Publications, May 2012 